\title{
¿CÓMO MIDE EL RIESGO EL OBSERVADOR IMPARCIAL?
}

\author{
Antonio J. Heras \\ Departamento de Economía Financiera y Contabilidad I \\ Universidad Complutense de Madrid \\ aheras@ccee.ucm.es \\ DAVID TEIRA \\ Departamento de Lógica, Historia y Filosofía de la Ciencia \\ UNED \\ dteira@fsof.uned.es
}

RESUMEN: Exploramos aquí la conexión entre los conceptos de riesgo e igualdad en el argumento del observador imparcial. La concepción de la justicia que elegiría un observador imparcial se justifica por la pureza del procedimiento de elección. Sin embargo, si modelizamos esta decisión utilizando medidas del riesgo habituales en matemática financiera, veremos cómo el criterio de elección del observador bajo el velo de la ignorancia contiene una preferencia implícita por el grado de desigualdad resultante. Esto nos obliga a reconsiderar la pureza procedimental de la elección.

PALABRAS CLAVE: posición original, procedimentalismo, incertidumbre, imparcialidad, medidas coherentes

SUMMARY: We study the link between the concepts of risk and equality in the argument of the impartial observer. The conception of justice that this latter would choose is grounded on the purity of the choice procedure. However, if we model this choice using the standard risk measurements in mathematical finance, there is an implicit preference for the degree of inequality that the chosen outcome will yield. This should make us reconsider the neutrality of the impartial observer's proceduralism.

KEY WORDS: original position, proceduralism, uncertainty, impartiality, coherent measures

\section{1. ¿Son normativamente neutrales nuestras representaciones del riesgo?}

El argumento del observador imparcial es uno de los más conocidos en la filosofía moral y política del siglo XX, tanto en el contractualismo rawlsiano, como en algunas versiones del utilitarismo (Harsanyi 1955, 1977a, 1977b, Broome 1991, Mongin 2001) o, más recientemente, en el suficientismo (Crisp 2003): privando al agente de cuanta información pudiese sesgar su elección, éste elegiría una organización social que sería procedimentalmente justa. Al cubrir así a nuestro agente con el velo de la ignorancia - la suya será una elección entre alternativas inciertas - , corre el riesgo de no obtener el resultado elegido. 
Es conocida la controversia entre Rawls y Harsanyi sobre cómo el observador imparcial habría de elegir bajo el velo de la ignorancia. Rawls defendió que debía aplicar el principio maximin: una distribución desigual de recursos sería justa en la medida en que maximizase el beneficio mínimo para los más desfavorecidos (aquellos con una dotación más baja de bienes primarios). Harsanyi, en cambio, optó por la teoría de la utilidad esperada, en la que el propio velo de la ignorancia era incorporado al criterio de decisión a través del postulado de equiprobabilidad: el observador imparcial debería elegir como si tuviera la misma probabilidad de ser uno de $\operatorname{los} n$ miembros de la sociedad. A partir de aquí, al observador de Harsanyi le bastaba con maximizar la utilidad esperada de su elección. Desde el punto de vista de Harsanyi (1975), el maximin era un criterio ya superado por la propia teoría de la decisión, pues hace depender la elección del observador imparcial de la peor de las contingencias sin importar qué probabilidad asignemos a que tal contingencia se produzca. Sin embargo, para Rawls el velo de la ignorancia traía consigo una incertidumbre radical: el observador imparcial no podía cuantificar probabilidades y de ahí su radical conservadurismo al elegir el maximin. Tal como Kurtulmus (2012) lo plantea, los velos de Rawls y de Harsanyi son de diferente grosor: el de Harsanyi es más fino, permite al menos cuantificar el riesgo (a través del postulado de equiprobabilidad); el de Rawls es más grueso, pues la incertidumbre es incuantificable.

En este artículo, asumimos, junto con Harsanyi (y contra Rawls) que el riesgo en la posición original es cuantificable, pero defenderemos que su cuantificación no es tan neutral normativamente como nos exigiría el velo de la ignorancia, al menos respecto a la igualdad de las distribuciones. Sin embargo, en la teoría de la utilidad esperada, riesgo y desigualdad son conceptos separados. El agente cuantifica el riesgo asignando una probabilidad a cada evento; sus preferencias sobre la igualdad o la desigualdad que estos eventos supongan se reflejan en su función de utilidad. Bajo el velo de la ignorancia, el observador imparcial debería plantearse la utilidad que una distribución de renta reportaría a $\operatorname{los} n$ individuos de una sociedad, ponderado por la probabilidad de obtener tal distribución. En virtud del postulado de equiprobabilidad, el observador imparcial tendría una probabilidad $1 / n$ de verse en la posición de cualquiera de los $n$ miembros de una sociedad. Así pues, al maximizar la utilidad esperada de la elección, nuestro observador elegiría la media aritmética de la utilidad de todas las posibles distribuciones. Así, el velo de la ignorancia serviría como garante de la justicia procedimental 
del reparto: a nuestro agente no se le podría acusar de escoger una distribución de la renta en función de sus intereses personales, sino conforme a una estimación positiva del riesgo $(1 / n)$.

La fuerza normativa de este argumento se basa, por lo tanto, en su carácter procedimental. La única característica psicológica que se supone en el observador es su grado de aversión al riesgo. En general, el grado de aversión al riesgo se corresponde con la forma de la función de utilidad. Para evaluar una distribución de valores $X=$ $\left(x_{1}, \ldots, x_{n}\right)$ (con probabilidades $\left(p_{1}, \ldots p_{n}\right)$, el decisor debe calcular la utilidad esperada mediante la expresión $E[u(X)]=u\left(x_{1}\right) \cdot p_{1}+\ldots+$ $u\left(x_{n}\right) p_{n}$. Cuando la función de utilidad es lineal, no hay aversión, es decir, hay indiferencia ante el riesgo; y será más cóncava cuanto mayor sea la aversión al riesgo.

Veamos un ejemplo sencillo. Supongamos que un agente debe elegir entre las distribuciones de renta $X=(1,2,3,4,5)$ e $Y=$ $(3,3,3,3,3)$, cuyos valores suponemos equiprobables. Si la función de utilidad con la que evalúa los resultados es $u(x)=x$ (lo que se corresponde con la indiferencia al riesgo del decisor), entonces la utilidad esperada de ambas distribuciones es la misma:

$$
\begin{gathered}
E(u(X))=u(1) \cdot(1 / 5)+u(2) \cdot(1 / 5)+u(3) \cdot(1 / 5)+u(4) \cdot(1 / 5)+u(5) \cdot(1 / 5)= \\
(1 / 5) \cdot(1+2+3+4+5)=3 \\
E(u(Y))=(1 / 5) \cdot(3+3+3+3+3)=3
\end{gathered}
$$

En este caso, la indiferencia al riesgo del decisor se traduce en una indiferencia a la desigualdad. Ahora bien, si la función de utilidad es cóncava y toma, por ejemplo, los valores $u(1)=1.0, u(2)=1.9$, $u(3)=2.7, u(4)=3.4, u(5)=4.0$, entonces los nuevos valores de la utilidad esperada son

$$
\begin{gathered}
E(u(X))=(1 / 5) \cdot(1+1.9+2.7+3.4+4)=13 / 5=2.6 \\
E(u(Y))=(1 / 5) \cdot(2.7+2.7+2.7+2.7+2.7)=2.7
\end{gathered}
$$

En este caso, la aversión al riesgo del observador hace que prefiera la distribución igualitaria $Y$ a la no igualitaria $X{ }^{1}$

\footnotetext{
${ }^{1}$ Este caso ilustra una propiedad general: si la función de utilidad es cóncava y por lo tanto el decisor tiene aversión al riesgo, se cumple que $E(u(X))<u(E(X))$,
} 
Para Rawls la aversión al riesgo sería moralmente irrelevante, pero para un utilitarista como Harsanyi $(1975$, p. 600; 1977a, p. 643) expresaría justamente la intensidad de las preferencias que una sociedad justa debiera satisfacer. Bajo el velo de la ignorancia, sin embargo, el principio de equiprobabilidad será independiente de nuestros intereses personales. Por lo tanto, la distribución elegida bajo el velo de la ignorancia será imparcial respecto a tales intereses y, en esa medida, puede ser objeto de acuerdo universal. ${ }^{2}$

La maximización de la utilidad esperada es, desde hace ya seis décadas, un modelo positivo de elección entre alternativas inciertas en múltiples ciencias sociales. En la teoría de la utilidad esperada la conexión entre riesgo e igualdad no es inmediatamente evidente: la forma de la función de utilidad puede usarse para medir la aversión al riesgo del observador, pero esto no se traduce automáticamente en una medida de la aversión a la desigualdad. La desigualdad es una propiedad relacional que tiene que ver con el grado en que los valores de la distribución se desvían o diferencian unos de otros, y esto no se tiene en cuenta en la expresión de la utilidad esperada $u\left(x_{1}\right) \cdot p_{1}+$ $\ldots u\left(x_{n}\right) \cdot p_{n}$, donde la aportación de cada valor $x_{i}$ es independiente de las aportaciones de los demás valores. Por ejemplo, la aportación a la utilidad esperada del valor $x_{5}=5$ en dos distribuciones equiprobables $(1,1,1,1,5)$ y $(5,5,5,5,5)$ es la misma: $(1 / 5) . u(5)$; pero esto contradice nuestra intuición básica sobre la desigualdad en ambas distribuciones, ya que en la primera distribución dicho valor es el "causante" de la desigualdad, algo que no sucede en la segunda.

En este artículo pretendemos mostrar, sin embargo, que al elegir una medida de riesgo optamos implícita o explícitamente por una

es decir, éste prefiere una distribución igualitaria cuyos valores coinciden con la esperanza matemática de la distribución $X$, a la propia distribución con riesgo $X$. Se puede encontrar una demostración de esta propiedad, así como de otros resultados de la teoría de la utilidad mencionados en el artículo, en el capítulo 6 de Mas-Colell et al. (1995).

${ }^{2}$ Es importante precisar que el observador imparcial de Harsanyi compara vectores que contienen las utilidades individuales de las distribuciones de riqueza (a los que aplica el postulado de equiprobabilidad), en lugar de vectores que contienen directamente las distribuciones de riqueza. El observador imparcial de Harsanyi es un ser racional (ya que sus decisiones se toman de acuerdo con las prescripciones de la teoría de la utilidad esperada), omnisciente (conoce las utilidades de todos los agentes, aunque no tiene necesariamente que ser uno de ellos) e imparcial (adopta el postulado de equiprobabilidad). Aunque las utilidades dependen a su vez de las distribuciones de riqueza, desde un punto de vista formal conviene distinguir ambas formulaciones. En este sentido, nuestra discusión no sigue fielmente el planteamiento de Harsanyi, sino que se debe considerar como una reelaboración o una simplificación. 
concepción normativa sobre la igualdad. En otras palabras, la elección de una medida de riesgo socavaría la pureza procedimental del argumento del observador imparcial.

Ilustremos intuitivamente, en primer lugar, la conexión entre riesgo y desigualdad. Desde un punto de vista puramente estadístico, existe cierta analogía formal entre ambos. Cuando hablamos, por ejemplo, del riesgo de una inversión suponemos que las probabilidades de obtener un beneficio se distribuyen conforme a una variable aleatoria. Cuando hablamos de desigualdad de la renta, suponemos que la distribución de la riqueza entre los miembros de una población no es uniforme. Riesgo y desigualdad se pueden cuantificar conforme a unas mismas distribuciones estadísticas (Pareto, lognormal, etc.) y esto permite apreciar una conexión intuitiva entre ambos conceptos: la distribución de probabilidad de los premios en una ruleta evidencia cuán arriesgada es una apuesta y cuán desigual será el reparto de los premios. Sin embargo, es evidente también que riesgo y desigualdad no se solapan por completo: mientras que las distribuciones desiguales a menudo se califican como justas o injustas, el riesgo suele considerarse normativamente neutro. Un inversor muy rico puede inspirar en muchos de nosotros ansias redistributivas, mientras que un inversor muy arriesgado nos parecerá, a lo sumo, excéntrico.

Sin embargo, en muchos contextos prácticos nos servimos de reglas de elección entre alternativas inciertas en las que el riesgo se maneja conforme a otros principios, como los que presentaremos en este artículo. Queremos explorar aquí, en particular, el enfoque de medición del riesgo desarrollado en los últimos quince años en el ámbito de las finanzas y los seguros. En lugar de partir de las preferencias subjetivas de los decisores sobre alternativas inciertas, nos centramos en ciertas características intuitivamente razonables que debería tener cualquier medida satisfactoria de un riesgo - por ejemplo, la necesidad de diversificarlo- - Al axiomatizar estas propiedades, aparecen distintos tipos de medidas del riesgo que permiten cuantificarlos numéricamente. La bibliografía sobre estas medidas y sus aplicaciones ha crecido exponencialmente en los últimos años. Una característica de estas medidas es que en ellas sí que se manifiesta de un modo explícito la conexión entre riesgo y desigualdad. Es más, nos permiten apreciar de qué modo está implícita esta conexión en la propia teoría de la utilidad esperada.

En este artículo queremos analizar cómo afectaría a la fuerza normativa del argumento del observador imparcial (en las versiones de Rawls y Harsanyi) su reformulación mediante conceptos alternativos 
de riesgo. Queremos poner de manifiesto cómo las distintas representaciones formales del riesgo que podamos utilizar al modelizar la elección del observador imparcial llevan implícita una preferencia normativa por el grado de desigualdad que resultará de la elección del observador imparcial. En este sentido, creemos que debemos reconsiderar el presunto carácter procedimental de concepto de justicia vehiculado a través del velo de la ignorancia.

Examinaremos en las dos secciones siguientes otras tantas concepciones alternativas del riesgo: cuando debemos elegir entre resultados inciertos podemos medir el riesgo según la variabilidad de estos resultados o según la magnitud de aquellos que nos supongan pérdidas. Presentaremos axiomáticamente, de modo informal, medidas de ambos conceptos (de coherencia y de desviación) habituales en matemática financiera. Sobre estas medidas presentaremos después funciones de decisión que nos permitirán construir modelos alternativos de la elección del observador imparcial. Estas medidas nos permitirán explicitar la conexión entre riesgo y desigualdad: si utilizamos un criterio de decisión basado en una medida coherente de riesgo para modelizar la elección del observador imparcial, podemos apreciar que nuestro observador elige implícitamente una medida de desviación que mide también el grado de desigualdad de las alternativas. Esto nos permitirá apreciar cómo en el procedimentalismo de Rawls y Harsnayi existe una preferencia implícita sobre la igualdad, lo cual pondría en duda la imparcialidad del observador.

\section{El riesgo como probabilidad de pérdida}

En general, una medida del riesgo es un funcional $\rho$ que asigna un número $\rho(X)$ a cada riesgo $X$, de forma que a mayor riesgo, mayor el valor de $\rho(X)$. Hay dos formas principales de definir el riesgo: en la primera acepción se dirá que una distribución con una gran variabilidad de resultados es arriesgada; en la segunda, diremos que a mayor probabilidad de obtener resultados desfavorables, mayor riesgo. Un billete de lotería tiene bastante riesgo del primer tipo (puede hacernos enormemente ricos), pero poco riesgo del segundo tipo (la pérdida máxima es el precio del billete, que suele ser pequeño). Parece intuitivamente claro que un agente interesado en evitar la desigualdad tenderá a usar la primera acepción de riesgo, mientras que un agente al que sólo le preocupa su propio bienestar preferirá la segunda. En ambos casos, una medida de riesgo debe obedecer al siguiente principio: la diversificación debe reducir el riesgo. Incluso antes de su axiomatización académica (Markowitz 1952), éste era sin duda el 
principio de gestión de riesgos que con más frecuencia encontramos en las más diversas actividades y culturas: las sociedades agrícolas diversifican los tipos de cultivo y los lugares donde plantan las cosechas, las sociedades ganaderas diversifican los tipos de ganado y los lugares de pastoreo y, en general, en los distintos mercados se observa cómo se intenta "no poner todos los huevos en la misma cesta" (Winterhalder et al. 1999). Las sociedades que no lo hacen suelen colapsar cuando cambian bruscamente las condiciones ambientales. El principio de diversificación no está convenientemente recogido en la modelización de los riesgos mediante funciones de utilidad, y ésta es precisamente una de las razones por las que se han introducido las modernas medidas del riesgo en la literatura reciente.

Si nos centramos en el riesgo definido como probabilidad de pérdida, las medidas del riesgo más populares entre los expertos son, claramente, las denominadas medidas coherentes (Artzner et al. 1999), caracterizadas por los siguientes axiomas:

Subaditividad: $\rho(x+y) \leq \rho(x)+\rho(y)$

Homogeneidad positiva: $\rho(c X)=c \rho(X), \forall c>0$

Invariancia por traslaciones: $\rho(X+c)=\rho(X)-c, \forall c \in \mathbb{R}$

Monotonicidad: $X(\omega) \leq Y(\omega), \forall \omega \in \Omega \Rightarrow \rho(X) \geq \rho(Y)$

En los axiomas anteriores, $X$ e $Y$ representan las distribuciones de bienes a las que hemos calificado anteriormente como riesgos, mientras que $\rho$ representa la medida del riesgo correspondiente. La propiedad de subaditividad representa matemáticamente la importancia de la diversificación: el riesgo de $X$ e $Y$ juntas es menor o igual que la suma de los riesgos por separado. Las propiedades de invariancia por traslaciones y de monotonicidad tienen sentido cuando se recuerda que estamos modelizando el riesgo concebido como posibilidad de pérdida, y por lo tanto las ganancias disminuyen el riesgo. La propiedad de homogeneidad positiva elimina el posible efecto distorsionador de los cambios en las unidades de medida.

Entre las medidas coherentes de riesgo, una de las más conocidas (por ser ampliamente utilizada en el sector financiero) es el denominado valor en riesgo condicional (Conditional Value at Risk: CVaR):

$$
\operatorname{CVaR}_{\varepsilon}(X)=\frac{1}{\varepsilon} \int_{0}^{\varepsilon} \operatorname{VaR}(X) d \varepsilon
$$


El $C V a R$ mide la pérdida esperada en caso de que las cosas vayan mal, ya que se puede demostrar que su valor coincide con (el opuesto de) la esperanza matemática condicionada a la cola desfavorable de la distribución, en la que hay acumulada una probabilidad $\varepsilon$ - de hecho, una denominación alternativa es esperanza condicionada de la cola, o Conditional Tail Expectation (CTE) —. El valor en riesgo $\operatorname{VaR}_{\varepsilon}$ es el (opuesto del) mínimo valor alcanzado con una probabili$\operatorname{dad} \varepsilon$ :

$$
\operatorname{VaR}_{\varepsilon}(X)=-\operatorname{Inf}_{x}\{x / P(X \leq x) \geq \varepsilon\}
$$

Es decir, $V a R$ es un cuantil de la distribución (cambiado de signo).

A modo de ilustración, supongamos la siguiente distribución de renta: un agente puede obtener las cantidades $(1,2,3,4,5)$. La renta esperada (la media de la distribución) es 3 . El valor en riesgo con un nivel de significación $\varepsilon=0.2$ es -1 : el valor mínimo que podemos obtener en la distribución es 1 ; su opuesto, según la definición de $V a R_{\varepsilon}$ es -1 , donde $\varepsilon=0.2$ es la probabilidad de obtener ese mínimo (un veinte por ciento de los casos, suponiendo que tengamos igual probabilidad de obtener cada uno de los cinco valores de la distribución). Si aumentamos la probabilidad $\varepsilon=0.4$, el $V a R_{\varepsilon}$ será -2 , pues $P(X \leq 2) \geq 0.4$. El valor en riesgo condicional $\mathrm{CVaR}$ con $\varepsilon=0.2$ también es -1 , y con $\varepsilon=0.4$ es -1.5 (la media entre -1 y -2 ). Es decir, gracias al VaR y al $\mathrm{CVaR}$ podemos estimar qué renta mínima nos corresponderá dependiendo de la probabilidad de que las cosas vayan mal.

Otra manera de generar medidas coherentes se basa en las llamadas funciones de distorsión (Wang 1996), usadas para modificar las probabilidades en un problema de decisión. Recordemos que la teoría de la utilidad esperada representa a los decisores adversos al riesgo mediante una función de utilidad cóncava. Una forma alternativa de representar la aversión al riesgo consiste en modificar las probabilidades, de forma que los eventos más desfavorables parezcan más probables de lo que realmente son. Dicha modificación se opera mediante una función de distorsión. Si esta función tiene ciertas propiedades (como ser no decreciente y cóncava), la esperanza matemática de la variable aleatoria bajo las probabilidades distorsionadas resulta ser una medida coherente. ${ }^{3}$

${ }^{3}$ Las medidas coherentes de distorsión pueden obtenerse también a partir de la teoría dual de la elección (Dual Theory of Choice) de Yaari (1987), y Tsanakas y Desli (2003). Para una panorámica de las teorías de la decisión alternativas a la utilidad esperada, véase Sugden 2004. 
Pues bien, recordemos aquí que el observador imparcial rawlsiano, bajo el velo de la ignorancia, tiene aversión radical al riesgo. ${ }^{4} \mathrm{La}$ intuición rawlsiana es que, al estar en juego la distribución de bienes primarios, su importancia justificaría una elección conservadora, independientemente de lo que cada quien decida sobre su vida ordinaria. Para Rawls apostar sobre, por ejemplo, nuestras libertades básicas, no tendría sentido. En cambio, una vez establecidas éstas, seremos libres de arriesgarnos cuanto queramos. Esta intuición rawlsiana se ha discutido ampliamente (Freeman 2012), y habría que admitirla aquí en aras de nuestro propio análisis. Si la aversión al riesgo es racional bajo el velo de la ignorancia - y obviamos por el momento la aversión rawlsiana a la cuantificación $-{ }^{5}$ la representación del riesgo más acorde con este criterio, de entre las examinadas en esta sección, será una medida coherente. Éstas garantizan al observador imparcial que si las utiliza se asegurará de que la distribución de los bienes primarios que elija minimizará sus pérdidas.

En cambio, desde una concepción distinta del velo de la ignorancia, Harsanyi defendió que, asumiendo la equiprobabilidad de ocupar cualquier posición social, el observador imparcial podía hacer uso de la teoría de la utilidad esperada y elegir entre concepciones de la justicia maximizando la utilidad social media. Como veremos en la siguiente sección, en ciertas condiciones, las medidas de coherencia nos permitirán también explicitar la conexión entre riesgo e igualdad en las decisiones del observador imparcial harsanyiano.

Por el momento, veamos cómo un observador imparcial que adoptase una medida de coherencia como criterio de elección entre distribuciones de renta ${ }^{6}$ debiera elegir bajo el velo de la ignorancia. Para modelizar esta elección, podemos definir una medida de preferencia $\Pi$ para nuestro observador como el valor de una medida coherente del riesgo cambiada de signo:

$$
\Pi(X)=-\rho(X)
$$

De esta forma, la condición de monotonía se transforma en:

Si $X(\omega) \leq Y(\omega)$, para todo escenario posible $\omega$,

${ }^{4}$ Aunque el propio Rawls (e.g., 1999, pp. 73, 144) advirtiera sobre la necesidad de diferenciar la aversión al riesgo en contextos ordinarios y en la posición original.

${ }^{5}$ Nos ocuparemos más detenidamente de este punto en la conclusión.

${ }^{6}$ Utilicemos la elección entre distribuciones de renta (en lugar de bienes primarios, etc.) en aras de la simplicidad. En realidad, la distribución de renta no forma parte de los bienes primarios a los que Rawls aplicaría su maximin. 


$$
\text { entonces } \Pi(X) \leq \Pi(Y)
$$

En otras palabras, si en una distribución siempre obtiene mejores resultados que en otra, en cualquier escenario posible, el observador imparcial elegirá la primera.

Si también la medida $\rho$ es además acotada por la media de la distribución de renta, resulta que

$$
\Pi(X)<E(X)
$$

siempre que $X$ no sea constante, dándose la igualdad entre ambos cuando $X$ es constante. Es decir, el observador valorará una distribución constante y sin riesgo precisamente en dicha cantidad constante (la esperanza de la distribución). En cambio, si la distribución no es constante, tendrá un riesgo para el observador, por lo que el valor de la función de preferencia será estrictamente menor que la esperanza de la distribución de renta. De hecho, esa valoración estará comprendida entre el menor valor de la distribución y su esperanza matemática: ${ }^{7}$

$$
\operatorname{Inf}(X) \leq \Pi(X) \leq E(X)
$$

Frente a este criterio de decisión, la teoría de la utilidad esperada estima nuestra preferencia por una distribución de renta según la esperanza matemática de obtenerla, suponiendo indiferencia al riesgo. La medida $\Pi$ corrige esta valoración teniendo en cuenta, además, el riesgo de la distribución. A mayor riesgo, más se alejará $\Pi$ de la esperanza matemática. Esto justifica que consideremos a $\Pi$ como una medida de eficiencia corregida por el riesgo. Por lo tanto, si el observador imparcial es adverso al riesgo, interpretado según una medida coherente, tratará de encontrar la distribución de renta que maximice $\Pi$. Veamos en la sección siguiente cómo profundizar en la conexión entre medidas de coherencia y utilidad esperada.

\footnotetext{
${ }^{7}$ Una medida de desviación $\delta$ se dice dominada inferiomente (lower range dominated) si para cualquier distribución $X$ verifica que $\delta(X) \leq E(X)-\operatorname{Inf}(X)$. Es decir, el valor de la medida es menor o igual que la esperanza de la distribución menos la mayor de sus cotas inferiores. Si $\rho$ es una medida coherente acotada por la media, entonces $\delta(X)=\rho(X-E(X))=\rho(X)+E(X)$ es una medida de desviación acotada inferiormente. Por lo tanto $\delta(X)=\rho(X)+E(X) \leq E(X)-\operatorname{Inf}(X)$, es decir, $\rho(X) \leq-\operatorname{Inf}(X)$ y $\Pi(X)=-\rho(X) \geq \operatorname{Inf}(X)$. La otra desigualdad es evidente a partir de la definición.
} 


\section{El riesgo como variabilidad}

Consideremos ahora la segunda forma de definir el riesgo a partir de la variabilidad: cuanto más amplio sea el rango de valores cubierto por una distribución, mayor probabilidad habrá de que nos toque uno adverso. Para formalizar este segundo concepto acudimos a las denominadas medidas de desviación (de ahora en adelante $\delta$ ), caracterizadas por las propiedades siguientes:

Subaditividad: $\delta(X+Y) \leq \delta(X)+\delta(Y)$

Homogeneidad positiva: $\delta(c X)=c \delta(X), \forall c>0$

Invariancia por traslaciones: $\delta(X+c)=\delta(X), \forall c \in \mathbb{R}$

No negatividad: $\delta(X)>0$ si $X$ no es constante, $\delta(X)=0$ si es constante.

Las dos primeras propiedades son las mismas que para las medidas coherentes. En particular, las medidas de desviación deben ser subaditivas y reconocer el valor de la diversificación, tal y como debe hacer toda medida del riesgo. La propiedad de invariancia por traslaciones implica que al modificar todos los valores de la distribución uniformemente por la misma cantidad, la medida de su variabilidad no cambia. La propiedad de no negatividad establece que la única distribución sin variabilidad es aquella en la que todos los valores coinciden en uno solo, la que podríamos denominar distribución constante.

Como ejemplos de medidas de desviación podemos mencionar la desviación típica y la desviación absoluta, así como las semidesviaciones (por la derecha y por la izquierda) correspondientes. La varianza, que es muy utilizada en la práctica como medida de dispersión, no es una medida de desviación en el sentido técnico que aquí utilizamos, ya que no es ni subaditiva ni homogénea.

No existe ninguna medida que sea a la vez coherente y de desviación, ya que las propiedades de invariancia por traslaciones para los dos casos son incompatibles entre sí. Existen, sin embargo, relaciones muy estrechas entre ambos tipos de medidas, siempre que añadamos propiedades adicionales a los conjuntos de axiomas que las definen. En Rockafellar et al (2006), se demuestra que a cada medida del riesgo coherente y acotada por la media se le puede asociar una única medida de desviación dominada inferiormente. ${ }^{8}$

${ }^{8}$ Véase la nota 4. En Rockafellar et al. (2006) se demuestra que toda medida coherente acotada por la media genera necesariamente una medida de desviación 
Pues bien, en el análisis de la posición original, asumimos que el observador imparcial no tiene otro interés que satisfacer sus preferencias, con independencia de las consecuencias que tuviera para los demás su elección. Supongamos ahora que el observador quiere incorporar explícitamente a su función de preferencia la desigualdad que su decisión puede generar. Para representar entonces el riesgo impuesto por el velo de la ignorancia será mejor ahora una medida de desviación, pues nuestro observador debe considerar ahora cuánta variabilidad está dispuesto a generar cuando opte por una distribución de renta.

De acuerdo con los resultados de Rockafellar et al. (2006), retomemos entonces $\Pi$ para generar una medida de desviación $\Delta$ de la siguiente manera:

$$
\Delta(X)=E(X)-\Pi(X)=E(X)+\rho(X)
$$

$\Delta$ medirá así el grado de desigualdad de la distribución comparando su esperanza matemática con la función de preferencia que en la sección anterior definimos sobre una medida coherente. Si queremos obtener una medida estandarizada cuyos valores varíen entre 0 (mínima desigualdad) y 1 (máxima desigualdad), podemos dividir $\Delta$ por su valor máximo $\mathrm{E}(\mathrm{X})$ y definir así el indice de desigualdad $I(X)$ :

$$
I(X)=\frac{\Delta(X)}{E(X)}=1-\frac{\Pi(X)}{E(X)}
$$

Las propiedades de nuestro índice son las siguientes: ${ }^{9}$

dominada inferiormente asociada con ella, y viceversa. La correspondencia viene dada por las siguientes expresiones:

Si se quiere obtener una medida de desviación $\delta$ a partir de una acotada por la media $\rho$,

$$
\delta(X)=\rho(X-E(X))=\rho(X)+E(X)
$$

Si se quiere obtener una medida acotada por la media $\rho$ a partir de una de desviación $\delta$,

$$
\rho(X)=E(-X)+\delta(X)
$$

${ }^{9}$ Todas las demostraciones son evidentes a partir de la definición del índice $I(X)$. Así, por ejemplo:

$$
\begin{aligned}
& I(X+c)=\frac{\Delta(X+c)}{E(X+c)}=\frac{\Delta(X)}{E(X+c)}=\frac{\Delta(X)}{E(X)+c}<\frac{\Delta(X)}{E(X)}=I(X) \\
& I(X+Y)=\frac{\Delta(X+Y)}{E(X+Y)} \leq \frac{\Delta(X)+\Delta(Y)}{E(X+Y)}=\frac{\Delta(X)+\Delta(Y)}{E(X)+E(Y)}= \\
& =\frac{\Delta(X)}{E(X)+E(Y)}+\frac{\Delta(Y)}{E(X)+E(Y)} \leq \frac{\Delta(X)}{E(X)}+\frac{\Delta(Y)}{E(Y)}=I(X)+I(Y)
\end{aligned}
$$




$$
\begin{gathered}
0 \leq I(X) \leq 1 \\
I(c X)=I(X) \\
I(X+C)<I(X) \\
I(C)=0, I(X)>0 \text { si } X \text { no es constante } \\
I(X+Y) \leq I(X)+I(Y)
\end{gathered}
$$

Las propiedades anteriores son razonables y muchas de ellas son bien conocidas en economía del bienestar. La segunda propiedad (invariancia de escala) establece que los cambios en las unidades de medida no modifican el grado de desigualdad. De acuerdo con la tercera, el reparto equitativo, en partes iguales, de bienes, reduce la desigualdad. La cuarta afirma que la desigualdad siempre es positiva, a menos que la distribución sea constante, es decir, que todos reciban la misma cantidad del bien.

¿Qué sucede entonces cuando el observador imparcial elige, bajo el velo de la ignorancia, apoyándose, pongamos, en $\Pi(X)$ ? Lo importante es que cuando el observador imparcial razona de acuerdo con una medida del riesgo $\rho$, es inevitable que implícitamente también razone de acuerdo con una medida de desviación $\Delta$ y una medida de desigualdad I (y viceversa). De hecho, si la esperanza es constante, la elección con respecto a I será la misma que la elección con respecto a $\rho$.

Por lo tanto, cuando el observador imparcial adopta $\Pi(X)$ como criterio de elección, el procedimiento sólo será imparcial respecto a sus intereses particulares, pero contiene una preferencia sustantiva sobre la desigualdad.

Retomemos el ejemplo anterior de la distribución de renta (1,2,3, $4,5)$, y supongamos que nuestro agente tiene preferencias rawlsianas, de modo que evalúa las distribuciones según los peores resultados posibles: deberá escoger, de entre las distribuciones que le ofrezcan, aquella en la que el riesgo de obtener el peor resultado sea menor. En nuestro ejemplo, el peor resultado posible es 1 , de modo que $\Pi(X)=$ 1. Las preferencias rawlsianas las genera una medida coherente del riesgo, el valor en riesgo condicional, con un nivel de significación 
igual a la probabilidad de obtener el peor resultado posible, que en nuestro ejemplo es el veinte por ciento. ${ }^{10}$

Como hemos visto anteriormente, estas preferencias inducen implícitamente una medida de desviación, y por lo tanto de desigualdad, $\Delta(X)=E(X)-\Pi(X)$. Como la esperanza de nuestra distribución es 3 , su desigualdad será $3-1=2$. Todo agente con preferencias rawlsianas evalúa, pues, la desigualdad de las distribuciones según la diferencia entre el valor medio y el peor valor. Para un agente rawlsiano, por lo tanto, las distribuciones $(1,2,3,4,5)$ y $(1,3,3,3,5)$ tienen el mismo grado de desigualdad. Intuitivamente, puede parecer que la segunda es más igualitaria que la primera, pero según la medida de desigualdad que emana de las preferencias rawlsianas no es así, ya que ambas tienen la misma medida $\Delta=2$ (y también el mismo índice de desigualdad I = 2/3). La medida rawlsiana de desigualdad es poco intuitiva porque también lo es la medida de preferencia que la origina.

Debemos, finalmente, poner de manifiesto la conexión entre las medidas de riesgo y la teoría de la utilidad esperada. Un resultado particularmente interesante es el siguiente: muchas medidas del riesgo coherentes, como el CVaR y las basadas en funciones de distorsión (dadas ciertas condiciones), son compatibles con la dominancia estocástica. ${ }^{11} \mathrm{Si}$ una distribución $X$ domina estocásticamente a otra

\footnotetext{
${ }^{10}$ Las preferencias rawlsianas también las puede generar el valor en riesgo, pero ésta no es una medida coherente.

${ }^{11}$ Dadas dos distribuciones $X$ e $Y$, se dice que $X$ domina a $Y$ según la dominancia estocástica de primer orden cuando sus funciones de distribución verifican que:
}

$$
F_{x}(x) \leq F_{Y}(x), \forall x \in \mathbb{R}^{+}
$$

Se puede demostrar que todo decisor maximizador de la utilidad esperada, con función de utilidad creciente, preferirá la distribución dominante $X$ en lugar de la distribución dominada $Y$. Se dice también que $X$ domina a $Y$ según la dominancia estocástica de segundo orden cuando sus funciones de distribución verifican que

$$
\int_{0}^{x} F_{x}(t) d t \leq \int_{0}^{x} F_{Y}(t) d t, \forall x \in \mathbb{R}^{+}
$$

Se puede demostrar que todo decisor maximizador de la utilidad esperada y adverso al riesgo, con función de utilidad creciente y cóncava, preferirá la distribución dominante $X$ en lugar de la distribución dominada $Y$. Todas las medidas coherentes del riesgo son "compatibles" con la dominancia estocástica de primer orden, lo que significa que, si $X$ domina a $Y$, entonces la medida del riesgo de $X$ debe ser menor o igual que la medida del riesgo de $Y$. Esto está garantizado por la propiedad de monotonía. Algunas medidas coherentes como el CVaR y las medidas de dispersión (bajo ciertas condiciones) son asimismo "compatibles" con la dominancia estocástica 
$Y$, todos los decisores que maximizan la utilidad esperada siendo adversos al riesgo prefieren $X$ a $Y$. Pues bien, en ese caso el riesgo de $X$ debe ser inferior al riesgo de $Y: \rho(X) \leq \rho(Y)$. Se verifica además que $\Pi(X) \geq \Pi(Y)$. Aplicándolo al caso que nos ocupa, si modelizamos la elección del observador imparcial mediante la teoría de la utilidad esperada, cuando todos los decisores maximizadores de la utilidad esperada y adversos al riesgo, con funciones de utilidad crecientes y cóncavas, prefieran $X$ a $Y$ obtendremos el mismo resultado que si lo modelizásemos con el CVaR.

\section{Es posible un procedimentalismo puro sobre el concepto de riesgo?}

Formulemos ahora explícitamente nuestra tesis: si utilizamos $\Pi$, un criterio de decisión basado en una medida coherente de riesgo, para modelizar la elección del observador imparcial, podemos apreciar que nuestro observador elige implícitamente una medida de desviación que mide también el grado de desigualdad de las alternativas. Al medir el riesgo y elegir bajo el velo de la ignorancia, el observador mide y elige también una dispersión de la distribución de renta resultante. Si recordamos los ejemplos mencionados en la introducción, es incluso intuitivo: al aumentar la desigualdad entre rentas, aumentan también las probabilidades de obtener un resultado desfavorable.

Por lo tanto, aun cuando el observador elija con independencia de su propia posición relativa en la distribución de rentas, el procedimiento (la representación de sus preferencias mediante una medida de coherencia) lleva implícita una inclinación sustantiva por minimizar la desigualdad. Un agente interesado exclusivamente en reducir su propio riesgo de ser pobre se verá así obligado a elegir de modo tal que se reduzca también el riesgo de que los demás sean pobres, aunque él mismo no sea en modo alguno altruista. En otras palabras, la elección de la medida de riesgo que nos permita, a su vez, elegir bajo el velo de la ignorancia es ya una elección moral, pues nos obliga a considerar ex ante la desigualdad que nuestra elección inducirá en la distribución de renta.

Recordemos, sin embargo, que tanto para Rawls como para Harsanyi la justicia como equidad se sostiene sobre un procedimen-

de segundo orden. Es decir, si $X$ domina a $Y$, entonces la medida del riesgo de $X$ debe ser menor o igual que la medida del riesgo de $Y$.

Dadas dos distribuciones arbitrarias, no siempre son comparables según los criterios de dominancia estocástica, por lo que el orden generado por estos criterios no es total sino parcial. Este hecho limita obviamente el rango de aplicaciones de estos conceptos. 
talismo puro, de modo tal que es el propio procedimiento lo que establece la justicia de la elección del observador imparcial. Es decir, individuos con distintas concepciones de la justicia debieran poder llegar a un acuerdo sobre la distribución de los bienes primarios, al cubrirse con el velo de la ignorancia. Su elección sería neutral respecto a sus intereses particulares y de ahí la justificación de su acuerdo. Pero si aceptamos que las medidas de coherencia son la opción más austera para modelizar la aversión al riesgo del observador imparcial, dado que no suponen en el agente ninguna preferencia por la igualdad, tenemos que admitir también que la pureza del procedimiento está viciada a favor del igualitarismo.

Aunque Rawls (2001, pp. xvii, 43, 95-96) sostuvo que la aversión al riesgo no contribuye a justificar el principio de diferencia, el análisis que proponemos muestra, de un modo nuevo, justamente lo contrario. Para Harsanyi (1975, p. 598), el postulado de equiprobabilidad se puede interpretar precisamente como expresión del principio moral de que el observador imparcial ha de concederle el mismo peso a priori a los intereses de todos los miembros de la sociedad. Pero, si nuestro análisis acierta, se diría que los igualitaristas tendrían los dados cargados a su favor.

Una réplica inmediata a nuestra objeción impugnará el uso de medidas del riesgo como las propuestas para modelizar la elección del observador imparcial. Al fin y al cabo, se alegará, en nuestro enfoque el observador imparcial elegiría con los mismos instrumentos de un corredor de bolsa. Nuestras medidas del riesgo son simples reglas de decisión que no captan toda la riqueza del razonamiento moral modelizado con la teoría de la utilidad esperada. La estructura de preferencias que la función $\Pi$ representa no nos permitiría analizar interacciones estratégicas, por ejemplo. Sin embargo, podría invertirse la objeción, pues hoy sabemos también, después de varias décadas de economía experimental, que las decisiones de los agentes económicos se desvían en numerosos contextos de la teoría de la utilidad esperada, sin que su justificación como canon normativo sean tampoco incontestables. Sin embargo, funciones de decisión como $\Pi$ son ampliamente utilizadas en toda clase de operaciones económicas, lo cual sugiere que, al menos como heurística para decisiones arriesgadas, las medidas de coherencia merecen consideración teórica. Además, como señalábamos al final de la sección anterior, el criterio de dominancia estocástica nos permite establecer cierta equivalencia entre medidas de riesgo y utilidad esperada.

Creemos que esta equivalencia nos ayuda a esclarecer parte, al menos, del éxito del argumento del observador imparcial. Pues, ¿es- 
taríamos dispuestos a aceptar una elección bajo el velo de la ignorancia que generase distribuciones extremadamente desiguales de la riqueza? Por ejemplo, dos distribuciones como $(10,10,10)$ y $(0,10,20)$ serían equivalentes para un decisor bajo el velo de la ignorancia e indiferente al riesgo. Pero la primera parece mucho más justa, en la medida en que es más igualitaria.

Un evaluador nos propone una objeción más global: si Rawls sostenía que bajo el velo de la ignorancia la incertidumbre era tan radical que no cabía cuantificación, al interpretar su argumento mediante medidas de riesgo no le somos fieles (como no lo será cualquier otra interpretación que trate de cuantificar semejante incertidumbre). Siendo esto cierto, cabe plantearse también nuestro argumento como un posible desarrollo de la posición de Rawls, que interesará al menos a quienes crean que su argumento sobre la incertidumbre radical no es defendible. Por ejemplo, Thomas Nagel (1973, p. 230) ya apuntó que en su Teoría de la justicia, Rawls justificaba semejante incertidumbre por la irracionalidad de apostar en una situación en la que los resultados (la distribución de bienes primarios) podían ser inaceptables. Pero, ¿por qué entonces no calcular probabilidades para todas las demás distribuciones por encima de ese umbral? Como Nagel indica, esto supondría poder comparar distribuciones que suponen ganancias para unos y pérdidas para otros - tal como haría, por ejemplo, un utilitarista-, lo cual a Rawls le resulta inaceptable. Pero impedir tales comparaciones, concluye Nagel, supone violar la imparcialidad procedimental de la posición original. Para remediarlo Kurtulmus (2012) propone un argumento sobre la imposibilidad de calcular probabilidades por el carácter inevitablemente vago de los términos que emplearíamos para efectuar comparaciones bajo el velo de la ignorancia.

En Justicia como equidad, el propio Rawls intenta aclarar el alcance de su argumento sobre la incertidumbre radical. ${ }^{12}$ Sin embargo, creemos que lo logra, una vez más, a costa del carácter procedimental de su argumento. Rawls (2001, p. 106) sostiene: en la posición original, "asumimos que la actitud de las partes ante la incertidumbre se basa en lo que consideran los intereses fundamentales de los ciu-

12 "Al argumentar a favor del principio de diferencia sobre otros principios distributivos [...] no se hace uso en absoluto de la regla maximin para decisiones bajo incertidumbre. La extendida idea de que el argumento a favor del principio de diferencia depende de la aversión al riesgo extrema es un error, desafortunadamente favorecido por los defectos de la exposición en Theory, que serán corregidos en la parte III de la presente reformulación (Rawls 2001, nota 43; la traducción es nuestra)." 
dadanos que representan". ${ }^{13}$ Es decir, la aversión al riesgo se basaría de nuevo en preferencias explícitamente morales. Por supuesto, como nuestro evaluador observa, podemos dar buenos argumentos para justificar estas preferencias, pero esto resta neutralidad procedimental a la posición original. ${ }^{14}$

Nuestro análisis nos permite mostrar, en cambio, que incluso si aceptáramos la posibilidad de cuantificar la incertidumbre, la aversión rawlsiana al riesgo lleva implícita una preferencia antiprocedimental por la igualdad, anterior al principio de diferencia. Dado el éxito del argumento de Rawls, podríamos pensar que semejante concepción del riesgo es ampliamente compartida. Desde este punto de vista, las medidas que acabamos de presentar pueden ayudarnos a reformular el argumento rawlsiano mediante un rango más extenso de preferencias sobre riesgo e igualdad, que ayudaran a definir con mayor precisión el alcance del contractualismo procedimentalista. Dejamos esta tarea para otra ocasión. ${ }^{15}$

\section{BIBLIOGRAFÍA}

Artzner, P., F. Delbaen, J. Eber, y D. Heath, 1999, "Coherent Measures of Risk", Mathematical Finance, vol. 9, no. 3, pp. 203-228.

Broome, J., 1991, Weighing Goods, Basil Blackwell, Oxford.

Crisp, R., 2003, "Equality, Priority and Compassion", Ethics, vol. 113, no. 4 , pp. $745-763$.

Freeman, S., 2013, Original Position, Stanford Encyclopedia of Philosophy, 2012 [fecha de consulta: 18/7/2013].

Harsanyi, J.C., 1977a, "Morality and the Theory of Rational Behavior", Social Research, vol. 44, no. 4, pp. 623-656.

—_ 1977b, Rational Behaviour and Bargaining Equilibrium in Games and Social Situations, Cambridge University Press, Cambridge.

_ 1975, "Can the Maximin Principle Serve as a Basis for Morality? A Critique of John Rawls's Theory", The American Political Science Review, vol. 69, no. 2, pp. 594-606.

— , 1955, "Cardinal Welfare, Individualistic Ethics, and Interpersonal Comparisons of Utility", Journal of Political Economy, vol. 63, no. 4, pp. 309-321.

${ }^{13}$ La traducción es nuestra.

${ }^{14}$ Agradecemos los apuntes de A. Faik Kurtulmus (en comunicación personal) sobre este punto.

${ }^{15}$ Los autores quieren agradecer los comentarios de A. Faik Kurtulmus, P.-Ch. Pradier y dos evaluadores anónimos.

La contribución de David Teira se inscribe en el proyecto de investigación FFI2011-28835. 
Harsanyi, J.C., 1953, "Cardinal Utility in Welfare Economics and in the Theory of Risk-Taking", Journal of Political Economy, vol. 61, pp. 434435.

Kurtulmus, A. Faik, 2012, "Uncertainty behind the Veil of Ignorance", Utilitas, vol. 24, no. 01, pp. 41-62.

Markowitz, H., 1952, "Portfolio Selection", The Journal of Finance, vol. 7, no. 1, pp. 77-91.

Mas-Colell, A., M. Whinston y J. Green, 1995, Microeconomic Theory, Oxford University Press, Nueva York.

Mongin, P., 2001, "The Impartial Observer Theorem of Social Ethics", Economics and Philosophy, vol. 17, no. 2, pp. 147-179.

Nagel, T., 1973, "Rawls on Justice", The Philosophical Review, vol. 82, no. 2, pp. 220-234.

Rawls, John, 2001, Justice as Fairness: A Restatement, Harvard University Press, Cambridge, Mass.

- 1999, A Theory of Justice, ed. revisada, Belknap Press of Harvard University Press, Cambridge, Mass.

Rockafellar, T., S. Uryasev, y M. Zabarankin, 2006, “Generalized Deviations in Risk Analysis", Finance and Stochastics, vol. 10, no. 1, pp. 51-74.

Sugden, R., 2004, "Alternatives to Expected Utility: Foundations", en P. Hammond, S. Barberá, y C. Seidl (eds.), Handbook of Utility Theory Vol 2: Extensions, Kluwer Academic Publishers, Dordrecht.

Tsanakas, A. y E. Desli, 2003, "Risk Measures and Theories of Choice", British Actuarial Journal, vol. 9, no. 4, pp. 959-991.

Wang, S., 1996, "Premium Calculation by Transforming the Layer Premium Density", ASTIN Bulletin, vol. 26, no. 1, pp. 71-92.

Winterhalder, B., F. Lu, y B. Tucker, 1999, "Risk-Sensitive Adaptive Tactics: Models and Evidence from Subsistence Studies in Biology and Anthropology", Journal of Archaeological Research, vol. 7, no. 4, pp. 301-348.

Yaari, M., 1987, "The Dual Theory of Choice under Risk", Econometrica, vol. 55, no. 1 , pp. 95-115.

Recibido el 8 de noviembre de 2013; revisado el 26 de octubre de 2014; aceptado el 13 de enero de 2015. 